\title{
Anticancer effect of icaritin inhibits cell growth of colon cancer through reactive oxygen species, Bcl-2 and cyclin D1/E signaling
}

\author{
CHAOFENG LI ${ }^{1}$, WEICHAO PENG ${ }^{2}$, XIN SONG $^{1}$, QIAN WANG ${ }^{3}$ and WENYUE WANG ${ }^{1}$ \\ Departments of ${ }^{1}$ Gastrointestinal Surgery and ${ }^{2}$ Breast and Thyroid Surgery, China-Japan Friendship Hospital; \\ ${ }^{3}$ Department of Cardiology, Beijing Anzhen Hospital, Beijing 100029, P.R. China
}

Received May 24, 2015; Accepted August 9, 2016

DOI: $10.3892 / 01.2016 .5089$

\begin{abstract}
Icaritin has an advantage in enhancing immunity. Besides, with its anticancer effect, it may be of great help in cancer treatment and recovery of cancer patients. As a result, icaritin is likely to become a novel anticancer drug. However, the anticancer effect of icaritin against colon cancer has not been elucidated thus far. The present study investigated the latent anticancer effect of icaritin on the inhibition of colon cancer cell growth by regulating reactive oxygen species (ROS), B-cell lymphoma (Bcl)-2 and cyclin D1/E signaling. The COLO-205 colon cancer cell line was used as a colon cancer cell model in the present study. First, cell growth and apoptosis were measured to analyze the anticancer effect of icaritin against colon cancer. Next, the possible mechanism of icaritin against colon cancer, including ROS, Bcl-2, cyclin D1, cyclin $\mathrm{E}$ and caspase-3/9, was explored. The results revealed that icaritin could inhibit cell growth and induce the apoptosis of COLO-205 cells. In addition, icaritin significantly induced ROS generation, suppressed Bcl-2, cyclin D1 and cyclin E protein expression, and activated caspase-3/9 activity in COLO-205 cells. The present findings demonstrated that icaritin exerted antiproliferative and anticancer effects against colon cancer through the activation of ROS generation and the suppression of Bcl-2, cyclin D1 and cyclin E signaling.
\end{abstract}

\section{Introduction}

Colon cancer is the world's third most common malignant tumor (1). In 2002, only in Europe, $>370,000$ people suffered from it, and $\sim 200,000$ people succumbed to the disease, which accounted for $12 \%$ of all cancer mortalities (2). In China, at present, colon cancer is ranked as the fifth most common tumor, whose growth speed is very fast, particularly in certain large cities (3). However, colon cancer's lack of specificity

Correspondence to: Mr. Wenyue Wang, Department of Gastrointestinal Surgery, China-Japan Friendship Hospital, 2 Yinghua Dongjie, Hepingli, Beijing 100029, P.R. China

E-mail:wangwy1988@yeah.net

Key words: icaritin, colon cancer, ROS, Bcl-2, cyclin D1, cyclin E in terms of clinical symptoms leads to patients being often misdiagnosed, thus delaying treatment (4). Therefore, research has focused on early detection and treatment of the tumor (5). The occurrence and development of colon cancer are associated with numerous factors, including activation of a variety of oncogenes and deactivation of tumor-suppressor genes, which are important in these processes $(5,6)$.

Induction of cell apoptosis is the main type of tumor treatment (7). Regulation of the intracellular redox state determines the cell's survival fate (8). In normal cells, two redox systems exist, the peroxide enzyme system and resistance against superoxide (9). The former includes the oxygen reduction sulfur protein, while the latter includes superoxide dismutase, catalase and glutathione peroxidase (10). These systems can remove reactive oxygen species (ROS) produced in the process of metabolism, which results in relatively stable levels of intracellular ROS (11). Multiple studies have confirmed that the level of intracellular ROS and the redox state are closely associated with cell apoptosis (12).

By far, the B-cell lymphoma (Bcl)-2 gene family is the most important gene family involved in the regulation of apoptosis (13). The family is divided into two categories: The first one comprises antiapoptotic proteins, mainly Bcl-2, Bcl-extra large, Bcl-W, A1/Bfl-1, myeloid cell leukemia 1 and Boo, while the second one comprises proapoptotic proteins, including Bcl-2 associated X protein (Bax), Bcl-2 homologous antagonist/killer, $\mathrm{Bcl}-2$ related ovarian killer, $\mathrm{Bcl}-\mathrm{x}, \mathrm{BH} 3$ interacting-domain death agonist and Bcl-2-associated death promoter (14). The Bcl-2 protein can inhibit cell apoptosis, but is unable to promote cell proliferation, in cancer cells (13).

Pharmacological studies have demonstrated that the active ingredients of icaritin can inhibit the proliferation of HL-60 and U-937 leukemia cells (15). Previous studies identified that the mechanism of inhibition of cell proliferation is mainly due to the induction of apoptosis and inhibition of telomerase activity (16). Icaritin was demonstrated to be able to alter the cell cycle of HL-60 cells (15). In addition, by increasing p21 tumor-suppressor genes and reducing c-Myc oncogene expression, antitumor effects were achieved (17). Previous reports have also demonstrated that icaritin's active ingredients can induce G1 phase retardation in prostate cancer cells, and cell growth was significantly inhibited as a consequence (17). Extensive research demonstrated that the active ingredients of icaritin can alter the extracellular signal-regulated kinase 
signaling pathway of liver cancer cells $(16,18)$. All the above results confirmed that icaritin has certain antitumor effects. The present study demonstrated that the anticancer effect of icaritin inhibits the growth of colon cancer cells through ROS, Bcl-2 and cyclin D1/E signaling.

\section{Materials and methods}

Chemicals and reagents. Dulbecco's modified Eagle's medium (DMEM), fetal bovine serum (FBS), 1-(4,5-dimethylthiazol2-yl)-3,5-diphenyl formazan (MTT) and icaritin (whose chemical structure is indicated in Fig. 1) were provided by Sigma-Aldrich (Merck Millipore, Darmstadt, Germany). Propidium iodide (PI) and the Annexin V-FITC Apoptosis Detection kit were purchased from BD Biosciences (Franklin Lakes, NJ, USA). The DC Protein Assay was purchased from Bio-Rad Laboratories, Inc. (Hercules, CA, USA).

Cell lines. The human colon cancer cell line COLO-205 was provided by the experimental center of China-Japan Friendship Hospital (Beijing, China). Cells were cultured in DMEM with $10 \%$ FBS, supplemented with $100 \mathrm{U} / \mathrm{ml}$ penicillin $\mathrm{G}$ and $100 \mu \mathrm{g} / \mathrm{ml}$ streptomycin (Gibco; Thermo Fisher Scientific, Inc., Waltham, MA, USA) at $37^{\circ} \mathrm{C}$ and $5 \% \mathrm{CO}_{2} / 95 \%$ air atmosphere.

Cell viability assay. COLO-205 cells were seeded at a density of $1-2 \times 10^{3}$ cells/well in a 96-well plate and cultured with icaritin $(0,1,2.5,5,7.5$ and $10 \mu \mathrm{M})$ for 1 or 2 days (19). Subsequently, MTT reagent was added to a final concentration of $0.5 \mathrm{mg} / \mathrm{ml}$ to each well, and the cells were cultured for additional $5 \mathrm{~h}$ at $37^{\circ} \mathrm{C}$ and $5 \% \mathrm{CO}_{2} / 95 \%$ air atmosphere. Upon incubation, the medium was removed, and $100 \mu 1$ dimethyl sulfoxide was added into each well. The absorbance was then read at $570 \mathrm{~nm}$ in a microplate reader (Molecular Devices, LLC, Sunnyvale, CA, USA).

Apoptosis assay. COLO-205 cells were seeded at a density of $1-2 \times 10^{6}$ cells/well in a 6 -well plate and cultured with icaritin $(0,1,5$ and $10 \mu \mathrm{M})$ for 2 days. Subsequently, the cells were collected with fluorescence-activated cell sorting wash buffer (BD Biosciences) and stained with annexin V-fluorescein isothiocyanate and PI (BD Biosciences) according to the manufacturer's protocol. Cell apoptosis was analyzed by flow cytometry (Accuri ${ }^{\mathrm{TM}} \mathrm{C} 6$; BD Biosciences).

ROS level detection. COLO-205 cells were seeded at a density of $1-2 \times 10^{6}$ cells/well in a 6-well plate and cultured with icaritin $(0,1,5$ and $10 \mu \mathrm{M})$ for 2 days. Subsequently, the cells were incubated with $50 \mu \mathrm{M} \mathrm{2}$ ',7'-dichlorofluorescein-diacetate (Sigma-Aldrich; Merck Millipore) for $10 \mathrm{~min}$ at $37^{\circ} \mathrm{C}$ and $5 \% \mathrm{CO}_{2} / 95 \%$ air atmosphere. ROS level was detected with a FACSCanto $^{\mathrm{TM}}$ flow cytometer (BD Biosciences).

Western blot assay. COLO-205 cells were seeded at a density of $1-2 \times 10^{6}$ cells/well in a 6-well plate and cultured with icaritin $(0,1,5$ and $10 \mu \mathrm{M})$ for 2 days. Subsequently, the cells were collected with Cell Lysis Buffer (Cell Signaling Technology, Inc., Danvers, MA, USA) by plate scraping on ice. Protein concentrations were evaluated by DC Protein Assay. A total of $20 \mathrm{mg}$ of protein was resolved on a $12 \%$ sodium dodecyl sulfate-polyacrylamide gel electrophoresis and then transferred to polyvinylidene fluoride membranes (GE Healthcare Life Sciences, Chalfont, UK). Membranes were blocked with 10\% non-fat dry milk in Tris-buffered saline with Tween 20 (TBST) for $1 \mathrm{~h}$ at room temperature, prior to be incubated with primary rabbit anti-human antibodies against Bcl-2 (1:1,000; sc-492; Santa Cruz Biotechnology, Inc., Dallas, TX, USA), cyclin D1 (1:500; sc-753; Santa Cruz Biotechnology, Inc.), cyclin E (1:2,000; sc-481; Santa Cruz Biotechnology, Inc.) and $\beta$-actin (1:2,000; sc-130656; Santa Cruz Biotechnology, Inc.) in TBST with $5 \%$ bovine serum albumin at $4^{\circ} \mathrm{C}$ overnight, followed by incubation with a peroxidase-conjugated secondary antibody (D110056; Sangon Biotech Co., Ltd., Shanghai, China) according to the manufacturer's protocol. Detection was performed with a highly sensitive chemiluminescent detection kit (C500044; Sangon Biotech Co., Ltd.).

Measurement of caspase-3 activity. COLO-205 cells were seeded at a density of $1-2 \times 10^{6}$ cells/well in a 6-well plate and cultured with icaritin $(0,1,5$ and $10 \mu \mathrm{M})$ for 2 days. Subsequently, the cells were collected with Cell Lysis Buffer by plate scraping on ice. Protein concentrations were evaluated by DC Protein Assay. Equal quantities of protein were added to the reaction buffer containing Asp-Glu-Val-Asp-p-nitroaniline, and were then incubated at $37^{\circ} \mathrm{C}$ for $6 \mathrm{~h}$ at room temperature. Caspase-3 activity was measured at an absorbance of $405 \mathrm{~nm}$ in a microplate reader (Molecular Devices, LLC).

Statistical analysis. Data are represented as the mean \pm standard error of the mean. Statistical analysis was conducted using with SPSS 11.5 statistical software (SPSS, Inc., Chicago, IL, USA) with the paired samples $t$-test. $\mathrm{P}<0.05$ was considered to indicate a statistically significant difference.

\section{Results}

Anticancer effect of icaritin on cell growth in COLO-205 cells. The anticancer effect of icaritin on cell growth was initially evaluated in COLO-205 cells using a standard MTT assay. Compared with COLO-205 cells without icaritin treatment, the cell growth of treated COLO-205 cells was inhibited in a dose- and time-dependent manner (Fig. 2). The inhibition of cell growth was significant upon treatment with 5, 7.5 and $10 \mu \mathrm{M}$ icaritin for 1 day, or with $2.5,5,7.5$ and $10 \mu \mathrm{M}$ for 2 days (Fig. 2).

Anticancer effect of icaritin on cell apoptosis in COLO-205 cells. The apoptosis of COLO-205 cells was analyzed using PI and the Annexin V-FITC Apoptosis Detection kit. Upon treatment with 5 and $10 \mu \mathrm{M}$ icaritin for 2 days, cell apoptosis was significantly increased, compared with that of COLO-205 cells without icaritin treatment (Fig. 3).

Anticancer effect of icaritin on ROS level in COLO-205 cells. To explore the underlying mechanisms regulating the anticancer effect of icaritin on the ROS level of COLO-205 cells, the ROS level was measured in different groups of COLO-205 cells. The ROS level was significantly enhanced following 5and $10-\mu \mathrm{M}$ icaritin treatment for 2 days in COLO-205 cells (Fig. 4). 
<smiles>COc1ccc(-c2oc3c(CC=C(C)C)c(O)cc(O)c3c(=O)c2O)cc1</smiles>

Figure 1. Chemical structure of icaritin.

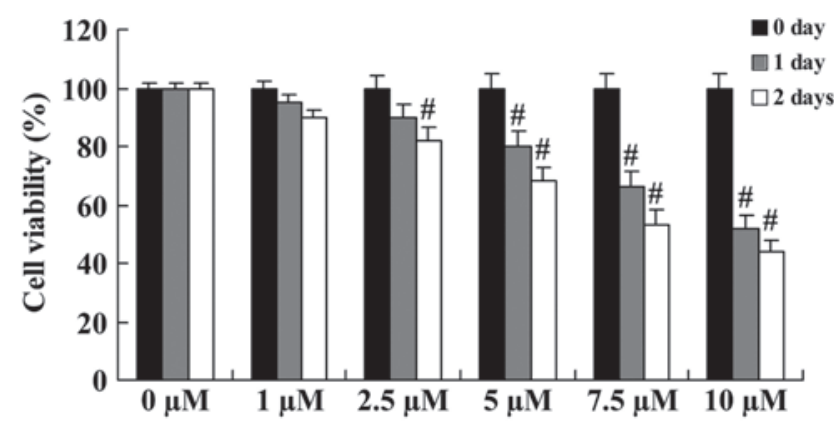

Figure 2. Anticancer effect of icaritin on cell growth in COLO-205 cells. ${ }^{\text {"}} \mathrm{P}<0.01$ compared with $0-\mu \mathrm{M}$ icaritin-treated group.

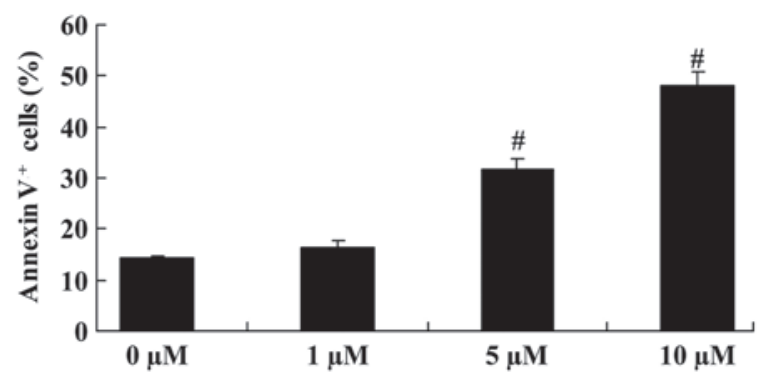

Figure 3. Anticancer effect of icaritin on cell apoptosis in COLO-205 cells. ${ }^{\#} \mathrm{P}<0.01$ compared with $0-\mu \mathrm{M}$ icaritin-treated group.

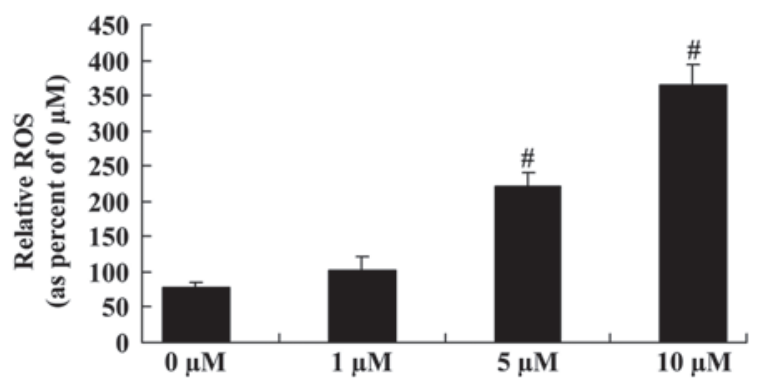

Figure 4. Anticancer effect of icaritin on ROS level in COLO-205 cells. ${ }^{\text {"}} \mathrm{P}<0.01$ compared with $0-\mu \mathrm{M}$ icaritin-treated group. ROS, reactive oxygen species.

Anticancer effect of icaritin on Bcl-2 expression in COLO-205 cells. The anticancer effect of icaritin on COLO-205 cells, which is frequently suppressed by Bcl-2 expression, was further assessed in COLO-205 cells. The inhibition of Bcl-2 protein expression correlated with the 5- and $10-\mu \mathrm{M}$ icaritin-induced cell apoptosis of COLO-205 cells treated for 2 days (Fig. 5).
Anticancer effect of icaritin on cyclin Dl expression in COLO-205 cells. To determine the anticancer effect of icaritin on cyclin D1 expression in COLO-205 cells, cyclin D1 protein expression was analyzed using a western blot assay. The results suggested that cyclin D1 protein expression of COLO-205 cells was effectively reduced by treatment with 5 and $10 \mu \mathrm{M}$ icaritin (Fig. 6).

Anticancer effect of icaritin on cyclin E expression in COLO-205 cells. To further investigate whether cyclin E directly influences the anticancer effects of icaritin on COLO-205 cells, cyclin E protein expression was confirmed by western blot analysis. The initial results revealed that 5 and $10 \mu \mathrm{M}$ of icaritin significantly inhibited cyclin $\mathrm{E}$ protein expression, which may be important for the anticancer effects of icaritin on COLO-205 cells (Fig. 7).

Anticancer effect of icaritin on caspase-3 activity in COLO-205 cells. The anticancer effect of icaritin on caspase-3 activity was next assessed in COLO-205 cells. As represented in Fig. 8, a significant reduction in caspase-3 activity was observed in COLO-205 cells treated with 5 and $10 \mu \mathrm{M}$ icaritin, compared with vehicle control.

\section{Discussion}

As one of the most common malignant tumors of the digestive system, colon cancer is severely harmful to humans (1). In recent years, with the continuous improvement of people's living standards in China and a gradual westernized diet, the incidence of colon cancer has increased year by year, which is a matter of concern (20). Large-scale population census studies have demonstrated that, among people older than 35 years, the incidence of colon cancer is $24-32$ cases/100,000 individuals (4). However, colon cancer prognosis is not optimistic, and the 5-year survival rate is $\sim 50 \%$ (21). All these observations enabled colon cancer to become a hot topic in tumor research. The present results demonstrate that icaritin significantly inhibited cell growth and induced cell apoptosis of COLO-205 cells. In addition, recent studies suggested that icaritin induced cell apoptosis in human lung cancer cells (22), renal cell carcinoma (19) and human osteosarcoma cells (23). Collectively, these data indicate that icaritin may exert potent antitumor and proapoptotic effects on human colon cancer.

ROS is a general term for reactive oxygen compounds, which are produced in the process of biological aerobic metabolism in the form of $\mathrm{O}^{-2}, \mathrm{H}_{2} \mathrm{O}_{2}, \mathrm{HO}^{-}$and nitric oxide (10). Besides, it is important in cell apoptosis through various different pathways (24). Numerous studies have demonstrated that ROS is important for killing tumor cells in all types of living organisms $(25,26)$. Besides, it may act as a second messenger to regulate cell proliferation, differentiation and apoptosis, which are associated with signal transduction pathways (10). A previous study has demonstrated that the antioxidant enzyme activity within the tumor cells was lower than that within normal cells, and that the removal efficiency of ROS was also low in tumor cells, suggesting that the intracellular ROS levels are more likely to kill tumor cells (27). Furthermore, recent studies have demonstrated that the excess of ROS in the cell can kill 
A

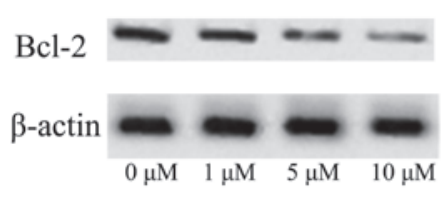

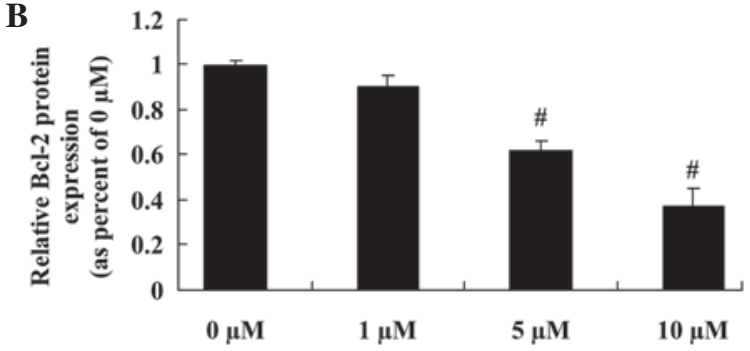

Figure 5. Anticancer effect of icaritin on Bcl-2 expression in COLO-205 cells. (A) Anticancer effect of icaritin on Bcl-2 expression by western blotting analysis and (B) statistical analysis of Bcl-2 expression in COLO-205 cells. ${ }^{*} \mathrm{P}<0.01$ compared with 0- $\mu \mathrm{M}$ icaritin-treated group. Bcl-2, B-cell lymphoma-2.
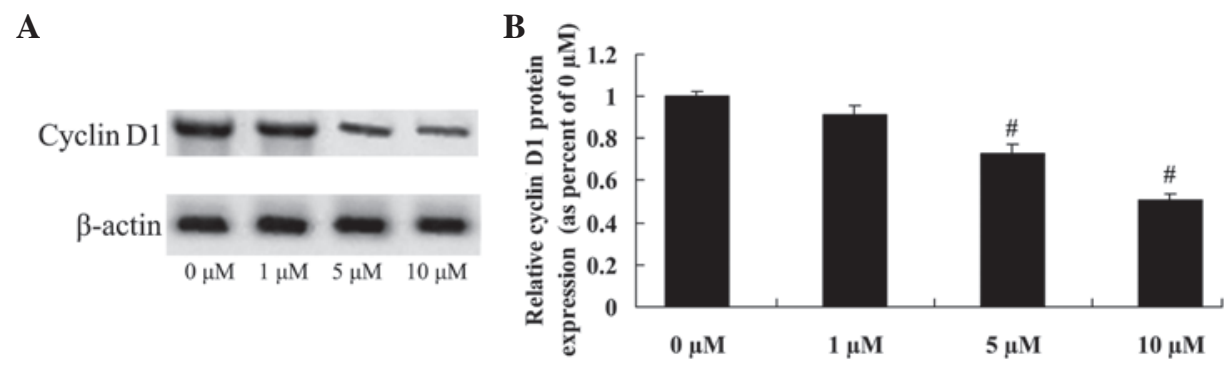

Figure 6. Anticancer effect of icaritin on cyclin D1 expression in COLO-205 cells. (A) Anticancer effect of icaritin on cyclin D1 expression by western blotting analysis and (B) statistical analysis of cyclin D1 expression in COLO-205 cells. ${ }^{*} \mathrm{P}<0.01$ compared with $0 \mu \mathrm{M}$-icaritin-treated group.

A

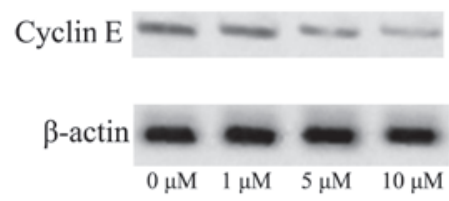

B

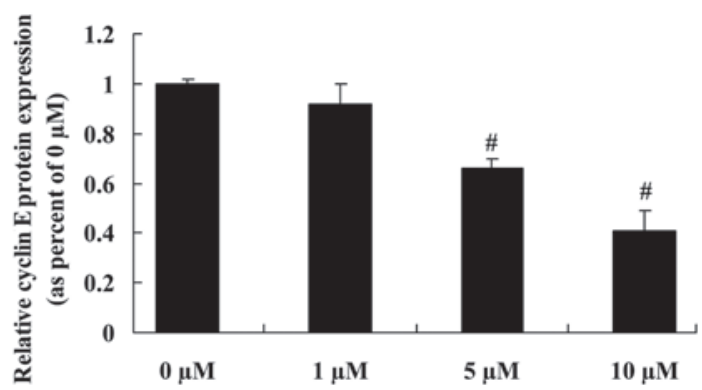

Figure 7. Anticancer effect of icaritin on cyclin E expression in COLO-205 cells. (A) Anticancer effect of icaritin on cyclin E expression by western blotting analysis and (B) statistical analysis of cyclin E expression in COLO-205 cells. ${ }^{*} \mathrm{P}<0.01$ compared with $0-\mu \mathrm{M}$ icaritin-treated group.

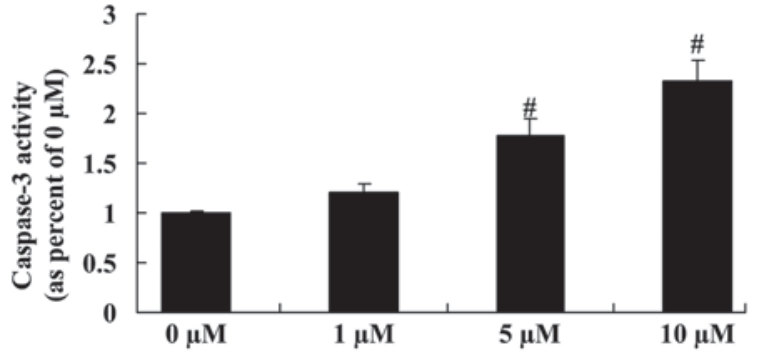

Figure 8. Anticancer effect of icaritin on caspase-3 activity in COLO-205 cells. ${ }^{\#} \mathrm{P}<0.01$ compared with $0-\mu \mathrm{M}$ icaritin-treated group.

tumor cells, thus suggesting a function for ROS on the treatment of tumors (12). In the present study, it was observed that icaritin treatment enhanced the ROS level of COLO-205 cells. Zheng et al suggested that icaritin induced the apoptosis of lung cancer cells through increasing their ROS level (22). The current results demonstrated that icaritin also modestly promoted the ROS level of COLO-205 cells, which may be attributed to the different signaling pathways that mediate the anticancer effect of icaritin on human colon cancer.

The $\mathrm{Bcl}-2$ protein, which is encoded by the $\mathrm{Bcl}-2$ gene, can inhibit apoptosis and prolong the life of the cell, but cannot promote cell proliferation (13). The protein encoded by the Bax gene, together with other antiapoptotic proteins, can be formed during the formation of Bcl-2 heterologous dimers, with the function of antagonizing the function of $\mathrm{Bcl}-2$ in order to promote cell apoptosis (14). Besides, structural changes in various organelles and in the cytoplasmic membrane, particularly in the mitochondrial outer membrane, as well as changes in the interaction between the antiapoptotic Bcl-2 protein and the membrane, may cause the loss of antiapoptotic proteins responsible for apoptosis inhibition, resulting in the loss of function of these organelles and the release of several apoptosis-promoting factors, eventually leading to cell apoptosis (14). The present study demonstrated that icaritin inhibits Bcl-2 protein expression in COLO-205 cells. A previous study also reported that icaritin induces the apoptosis of $\mathrm{HepG} 2$ cells through modulating the $\mathrm{Bax} / \mathrm{Bcl}-2$ ratio (18) and that of breast 
cancer cells through the downregulation of Bcl-2 expression (16). In present study, icaritin significantly inhibited Bcl-2 expression in COLO-205 cells.

The normal growth of cells in the body depends on the balance of various regulatory factors involved in the regulation of the cell cycle. Therefore, any type of occurring disorder involving regulatory factors will lead to abnormal cell proliferation, thus inducing tumor formation and progression. Cyclin D1 can be combined with cyclin-dependent kinase 4 in the cell cycle to promote cell cycle progression, and therefore, is regarded as a type of proto-oncogene (28). Cyclin D1 protein expression is significantly correlated with colon cancer histology, presence of lymph node metastasis and staging, and colon cancer progression and metastasis (29).

Cyclin E has been confirmed to regulate the cell cycle in cancer cells. Besides, it is important in the process of tumor development. Previous studies have demonstrated that cyclin $\mathrm{E}$ is the main protein involved in mediating G1/S-phase transformation, thus being important in cell proliferation (30). Cyclin E expression can shorten the G1 phase of the cell cycle, cause centrosome proliferation, disrupt mitosis and lead to the formation of chromosome instability, thus inducing tumor formation (30). Cyclin E overexpression could increase the number of colon cancer cells in the G1/S phase. Furthermore, cyclin E overexpression has been associated with tumor progression and metastasis $(31,32)$. The present study revealed that icaritin effectively reduced cyclin D1 and cyclin E protein expression in COLO-205 cells. Li et al suggested that icaritin inhibits the cell growth of renal cell carcinoma through cyclin D1 and cyclin E (19).

In conclusion, to the best of our knowledge, the present study has reported for the first time that the anticancer effect of icaritin inhibits cell growth and induces apoptosis of human colon cancer. The underlying mechanisms of icaritin may be associated with the promotion of ROS, and the inhibition of Bcl-2 and cyclin D1/E signaling. The current study highlights icaritin as a potential anticancer target for treating metastatic cancer through the regulation of ROS, Bcl-2 and cyclin D1/E signaling.

\section{References}

1. Lee WS, Yun JW, Nagappan A, Park HS, Lu JN, Kim HJ, Chang SH, Kim DC, Lee JH, Jung JM, et al: Tetraarsenic hexoxide demonstrates anticancer activity at least in part through suppression of NF-kB activity in SW620 human colon cancer cells. Oncol Rep 33: 2940-2946, 2015.

2. Quaglia A, Tavilla A, Shack L, Brenner H, Janssen-Heijnen M, Allemani C, Colonna M, Grande E, Grosclaude P and Vercelli M; EUROCARE Working Group: The cancer survival gap between elderly and middle-aged patients in Europe is widening. Eur J Cancer 45: 1006-1016, 2009.

3. Chang W, Wei Y, Ren L, Zhong Y, Yu Y, Chen J, Zhu D, Ye L, Qin C, Zhao N, et al: Randomized controlled trial of intraportal chemotherapy combined with adjuvant chemotherapy (mFOLFOX6) for stage II and III colon cancer. Ann Surg 263: 434-439, 2016.

4. Aleksandrova K, Pischon T, Buijsse B, May AM, Peeters PH, Bueno-de-Mesquita HB, Jenab M, Fedirko V, Dahm CC, Siersema PD, et al: Adult weight change and risk of colorectal cancer in the European prospective investigation into cancer and nutrition. Eur J Cancer 49: 3526-3536, 2013.

5. Li F, Chen Z, Yang Y, Yi X, Yang Y and Zhang L: Characterization of the pathologic and endoscopic measurements of colorectal polyp sizes with a focus on sessile serrated adenoma and high-grade dysplasia. Int J Clin Exp Pathol 7: 1635-1643, 2014.
6. Sahm M, Wesselmann S, Kube R, Schöffel N, Pross M, Lippert H and Kahl S: The development process of colon cancer centres. Zentralbl Chir 138: 33-37, 2013 (In German).

7. Rivoltini L, Chiodoni C, Squarcina P, Tortoreto M, Villa A, Vergani B, Bürdek M, Botti L, Arioli I, Cova A, et al: TNF-related apoptosis-inducing ligand (TRAIL)-armed exosomes deliver proapoptotic signals to tumor site. Clin Cancer Res 22: 3499-3512, 2016.

8. Bhattacharyya S, Pal PB and Sil PC: A 35 kD Phyllanthus niruri protein modulates iron mediated oxidative impairment to hepatocytes via the inhibition of ERKs, p38 MAPKs and activation of PI3k/Akt pathway. Food Chem Toxicol 56: 119-130, 2013.

9. Arts WF, Scholte HR, Loonen MC, Przyrembel H, Fernandes J, Trijbels JM and Luyt-Houwen IE: Cytochrome c oxidase deficiency in subacute necrotizing encephalomyelopathy. J Neurol Sci 77: 103-115, 1987.

10. Araki K and Nagata K: Functional in vitro analysis of the ERO1 protein and protein-disulfide isomerase pathway. J Biol Chem 286: 32705-32712, 2011.

11. Satapathy SR, Mohapatra P, Das D, Siddharth S and Kundu CN: The apoptotic effect of plant based nanosilver in colon cancer cells is a p53 dependent process involving ROS and JNK cascade. Pathol Oncol Res 21: 405-411, 2015.

12. Peng $X$ and Gandhi V: ROS-activated anticancer prodrugs: A new strategy for tumor-specific damage. Ther Deliv 3: 823-833, 2012.

13. Zhou Y, Liu QH, Liu CL and Lin L: Calycosin induces apoptosis in human ovarian cancer SKOV3 cells by activating caspases and Bcl-2 family proteins. Tumour Biol 36: 5333-5339, 2015.

14. Alibek K, Irving S, Sautbayeva Z, Kakpenova A, Bekmurzayeva A, Baiken Y, Imangali N, Shaimerdenova M, Mektepbayeva D, Balabiyev A and Chinybayeva A: Disruption of Bcl-2 and Bcl-xL by viral proteins as a possible cause of cancer. Infect Agent Cancer 9: 44, 2014.

15. Li Q, Huai L, Zhang C, Wang C, Jia Y, Chen Y, Yu P, Wang H, Rao Q, Wang M and Wang J: Icaritin induces AML cell apoptosis via the MAPK/ERK and PI3K/AKT signal pathways. Int J Hematol 97: 617-623, 2013.

16. Guo Y, Zhang X, Meng J and Wang ZY: An anticancer agent icaritin induces sustained activation of the extracellular signal-regulated kinase (ERK) pathway and inhibits growth of breast cancer cells. Eur J Pharmacol 658: 114-122, 2011.

17. Tong JS, Zhang QH, Huang X, Fu XQ, Qi ST, Wang YP, Hou Y, Sheng J and Sun QY: Icaritin causes sustained ERK1/2 activation and induces apoptosis in human endometrial cancer cells. PLoS One 6: e16781,2011.

18. He J, Wang Y, Duan F, Jiang H, Chen MF and Tang SY: Icaritin induces apoptosis of HepG2 cells via the JNK1 signaling pathway independent of the estrogen receptor. Planta Med 76: 1834-1839, 2010.

19. Li S, Priceman SJ, Xin H, Zhang W, Deng J, Liu Y, Huang J, Zhu W, Chen M, Hu W, et al: Icaritin inhibits JAK/STAT3 signaling and growth of renal cell carcinoma. PLoS One 8: e81657, 2013

20. Vemulapalli R, Lara LF, Sreenarasimhaiah J, Harford WV and Siddiqui AA: A comparison of palliative stenting or emergent surgery for obstructing incurable colon cancer. Dig Dis Sci 55: 1732-1737, 2010

21. Haghighi MM, Vahedi M, Mohebbi SR, Pourhoseingholi MA, Fatemi SR and Zali MR: Comparison of survival between patients with hereditary non polyposis colorectal cancer (HNPCC) and sporadic colorectal cancer. Asian Pac J Cancer Prev 10: 209-212, 2009.

22. Zheng Q, Liu WW, Li B, Chen HJ, Zhu WS, Yang GX, Chen MJ and $\mathrm{He}$ GY: Anticancer effect of icaritin on human lung cancer cells through inducing $\mathrm{S}$ phase cell cycle arrest and apoptosis. J Huazhong Univ Sci Technolog Med Sci 34: 497-503, 2014.

23. Wang XF and Wang J: Icaritin suppresses the proliferation of human osteosarcoma cells in vitro by increasing apoptosis and decreasing MMP expression. Acta Pharmacol Sin 35: 531-539, 2014.

24. Trachootham D, Zhou Y, Zhang H, Demizu Y, Chen Z, Pelicano H, Chiao PJ, Achanta G, Arlinghaus RB, Liu J and Huang P: Selective killing of oncogenically transformed cells through a ROS-mediated mechanism by beta-phenylethyl isothiocyanate. Cancer Cell 10: 241-252, 2006.

25. Gong EY, Shin YJ, Hwang IY, Kim JH, Kim SM, Moon JH, Shin JS, Lee DH, Hur DY, Jin DH, et al: Combined treatment with vitamin $C$ and sulindac synergistically induces p53- and ROS-dependent apoptosis in human colon cancer cells. Toxicol Lett 258: 126-133, 2016 
26. Park KW, Kundu J, Chae IG, Bachar SC, Bae JW and Chun KS: Methanol extract of Flacourtia indica aerial parts induces apoptosis via generation of ROS and activation of caspases in human colon cancer HCT116 cells. Asian Pac J Cancer Prev 15: 7291-7296, 2014

27. Zhai W, Xu YF, Peng B, Zhang HM, Huang JH, Liu M, Wang GC and Zheng JH: Effect of free radical scavenger on c-jun activation in rats with crush syndrome. Int J Clin Pharmacol Ther 51: 600-605, 2013.

28. Gopalakrishnan N, Saravanakumar M, Madankumar P, Thiyagu $\mathrm{M}$ and Devaraj $\mathrm{H}$ : Colocalization of $\beta$-catenin with Notch intracellular domain in colon cancer: A possible role of Notch1 signaling in activation of CyclinD1-mediated cell proliferation. Mol Cell Biochem 396: 281-293, 2014.

29. Qin A, Yu Q, Gao Y, Tan J, Huang H, Qiao Z and Qian W: Inhibition of STAT3/cyclinD1 pathway promotes chemotherapeutic sensitivity of colorectal caner. Biochem Biophys Res Commun 457: 681-687, 2015
30. Prosnitz RG, Patwardhan MB, Samsa GP, Mantyh CR, Fisher DA, McCrory DC, Cline KE, Gray RN and Morse MA: Quality measures for the use of adjuvant chemotherapy and radiation therapy in patients with colorectal cancer: A systematic review. Cancer 107: 2352-2360, 2006.

31. Patwardhan MB, Samsa GP, McCrory DC, Fisher DA, Mantyh CR, Morse MA, Prosnitz RG, Cline KE and Gray RN: Cancer care quality measures: Diagnosis and treatment of colorectal cancer. Evid Rep Technol Assess (Full Rep): 1-116, 2006.

32. Rahmutulla B, Matsushita K, Satoh M, Seimiya M, Tsuchida S, Kubo S, Shimada H, Ohtsuka M, Miyazaki M and Nomura F: Alternative splicing of FBP-interacting repressor coordinates c-Myc, P27Kip1/cyclinE and Ku86/XRCC5 expression as a molecular sensor for bleomycin-induced DNA damage pathway. Oncotarget 5: 2404-2417, 2014. 